\title{
THÉORÈMES FONDAMENTAUX DE LA THÉORIE DES ÉQUATIONS DIFFÉRENTIELLES ORDINAIRES, II
}

\author{
Par
}

\section{Masuo HUKUHARA}

(Reçu le 16 janvier, 1940)

\section{TABLE DES MATIÊRES}

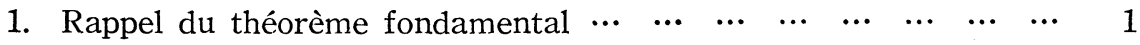

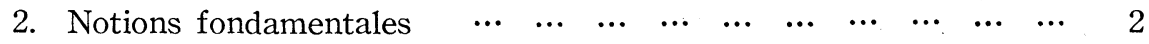



4. Comparaison à l'aide de la fonction de M. KAmke $\cdots \quad$...

5. Comparaison d'un système différentiel avec une seule équation $\begin{array}{lllllllllllllll}\text { différentielle } & \cdots & \ldots & \ldots & \ldots & \ldots & \ldots & \ldots & \ldots & \ldots & \ldots & \ldots & \ldots & \ldots & 12\end{array}$

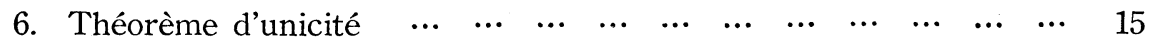

7. Continuité $\ldots \begin{array}{lllllllllllllll} & \ldots & \ldots & \ldots & \ldots & \ldots & \ldots & \ldots & \ldots & \ldots & \ldots & \ldots & \ldots & \ldots & 16\end{array}$

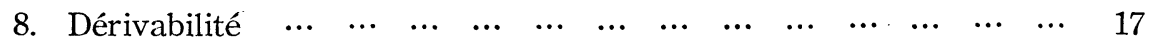

9. Analyticité $\begin{array}{llllllllllllllll} & \ldots & \ldots & \ldots & \ldots & \ldots & \ldots & \ldots & \ldots & \ldots & \ldots & \ldots & \ldots & \ldots & 23\end{array}$

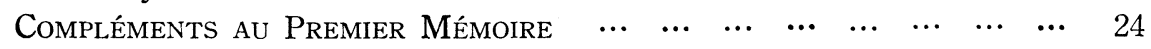

1. Rappel du théorème fondamental. Nous allons étendre, dans ce présent mémoire, les résultats du Premier Mémoire au cas d'un système d'équations différentielles à l'aide de la fonction $S$ de M. KaMKE.

Rappelons d'abord le théorème d'existence établi par moi $\left({ }^{1}\right)$. Nous désignons par $\vec{y}$ le point dont les coordonnées sont $y_{1}, y_{2}, \ldots$, $y_{n}$, de sorte que le système d'équations différentielles

$$
y_{j}^{\prime}=f_{j}\left(x, y_{1}, \ldots, y_{n}\right) \quad(j=1,2, \ldots, n)
$$

peut être représenté par une seule équation

（1）福原, 常微分方程式/基本定理, I, 日本數學物理學會誌, 5 (1931), 331-334. 


$$
\overrightarrow{y^{\prime}}=\vec{f}(x, \vec{y}) \text {. }
$$

Le théorème s'énonce alors comme il suit.

THÉORÈmE 1. Soit $E$ un ensemble de points (dans l'espace à $n+1$ dimensions) fermé sur un ensemble $D$ qui le contient. Soit $\vec{f}(x, \vec{y})$ une fonction continue dans $E$. Nous supposons de plus les hypothèses suivantes remplies:

$1^{\circ}$ A chaque point $\left(x_{0}, \vec{y}_{0}\right)$ de $E$, on peut faire correspondre un nombre positif $\delta$ tel que le point $(x, \vec{y})$, satisfaisant aux relations

$$
0 \leqq x-x_{0}<\delta,\left|\frac{\vec{y}-\overrightarrow{y_{0}}}{x-x_{0}}-\vec{f}\left(x_{0}, \overrightarrow{y_{0}}\right)\right|<\delta,
$$

appartient à $D$.

$2^{\circ}$ A chaque point $\left(x_{0}, \vec{y}_{0}\right)$ de $E$, on peut faire correspondre une fonction $\vec{\varphi}(x)$ continue dans l'intervalle $0 \leqq x-x_{0}<\delta$, contenue dan $E\left({ }^{2}\right)$ et satisfaisant aux relations

$$
\vec{\varphi}\left(x_{0}\right)=\overrightarrow{y_{0}}, \quad \lim _{x \rightarrow x_{0}+0} \frac{\vec{\varphi}(x)-\vec{y}_{0}}{x-x_{0}}=\vec{f}\left(x_{0}, \overrightarrow{y_{0}}\right) .
$$

Dans ces hypothèses, il existe au moins une solution de (1), continue dans un certain intervalle $x_{0} \leqq x<a$, contenue dans $E$ et satisfaisant à la condition initiale $\vec{y}\left(x_{0}\right)=\vec{y}_{0},\left(x_{0}, \vec{y}_{0}\right)$ désignant un point quelconque de $E$. Si l'on ne peut prolonger cette solution $\vec{y}(x)$ au delà de $x=a$, la suite de points $\left\{\left(a_{m}, \vec{y}\left(a_{m}\right)\right)\right\}$ ne peut converger en un point de $E$, de quelque manière qu'on choisisse la suite croissante $\left\{a_{m}\right\}$ tendant vers $a$.

2. Notions fondamentales. Nous introduisons maintenant quelques notions très utiles dans la suite.

DéFInITION 1 . Un ensemble $E$, contenu dans $D$, est dit majorant à droite en $D$, si la propriété suivante est vérifiée:

Une solution quelconque de (1), issue d'un point $\left(x_{0}, \overrightarrow{y_{0}}\right)$ de $E$, continue dans un intervalle $x_{0} \leqq x \leqq a$ et contenue dans $D$, est nécessairement contenue dans $E$.

(2) Cela veut dire que la courbe $\vec{y}=\vec{p}(x)$ est contenue dans $E$. 
Théorèmes Fondamentaux de la Théorie des Équations Dif.

On définira de la même manière ensemble majorant à gauche en $D$.

Les théorèmes suivants sont évidents.

THÉORÈmE 2. Si $E$ est majorant à droite en $D, D-E$ est majorant à gauche en $D$.

THÉORÈmE 3. La somme et le produit d'un nombre fini ou infini d'ensembles majorants à droite en $D$ sont aussi majorants à droite en $D$.

ThÉORÈme 4. Pour qu'un ensemble $E$ (contenu dans $D$ ) fermé sur $D$ soit majorant à droite en $D$, il suffit qu'il soit localement majorant à droite en $D\left({ }^{3}\right)$.

DÉFInITION 2. S'il existe un nombre positif $\delta$ tel que le point $(x, \vec{y})$, satisfaisant aux relations

$$
0 \leqq x-x_{0}<\delta,\left|\frac{\vec{y}-\vec{y}_{0}}{x-x_{0}}-\vec{f}\left(x_{0}, \overrightarrow{y_{0}}\right)\right|<\delta,
$$

appartient à $D$, l'ensemble $D$ est dit dominant à droite en $\left(x_{0}, \vec{y}_{0}\right)$. Si $D$ est dominant à droite en chaque point de $E, D$ est dit dominant à droite en $E$.

On définira de la même manière ensemble dominant à gauche en un point ou en un ensemble.

DÉfinition 3. ' S'il existe une fonction $\vec{\varphi}(x)$ continue dans un intervalle $0 \leqq x-x_{0}<\delta$, contenue dans $E$ et satisfaisant aux relations

$$
\vec{\varphi}\left(x_{0}\right)=\vec{y}_{0}, \lim _{x \rightarrow x_{0}+0} \frac{\vec{\varphi}(x)-\vec{y}_{0}}{x-x_{0}}=\vec{f}\left(x_{0}, \overrightarrow{y_{0}}\right),
$$

$D$ est dit quasi dominant $\grave{a}$ droite en $\left(x_{0}, \vec{y}_{0}\right)$. Si $D$ est quasi dominant à droite en chaque point de $E, D$ est dit quasi dominant à droite en $E$.

On définira de la même manière ensemble quasi dominant $\grave{a}$ gauche en un point ou en un ensemble.

En employant ces terminologies, nous pouvons énoncer les hypothèses $1^{\circ}$ et $2^{\circ}$ du théorème 1 comme il suit:

(3) Cela veut dire qu'à chaque point $\left(x_{0}, \vec{y}_{0}\right)$ de $E$ on peut faire correspondre un nombre positif $h$ tel qu'une solution quelconque, issue du point $\left(x_{0}, \overrightarrow{y_{0}}\right)$ et contenue dans $D$, est contenue dans $E$ pour $0 \leqq x-x_{0}<h$. 
$1^{\circ} \quad D$ est dominant à droite en $E$.

$2^{\circ} E$ est quasi dominant à droite en soi.

Les théorèmes suivants sont évidents.

THÉORÈME 5. Si $D_{k}$ est (quasi) dominant à droite en $E_{k}$, $\sum_{k} D_{k}$ est (quasi) dominant à droite en $\sum_{k} E_{k}$, l'indice $k$ pouvant prendre en nombre fini ou infini de valeurs.

THÉORÈME 6. Si $D_{k}$ est dominant à droite en $E_{k}(k=1,2, \ldots$, $m), \prod_{k=1}^{m} D_{k}$ est dominant à droite en $\prod_{k=1}^{m} E_{k}$.

THÉORÈME 7. Si $D_{1}$ est dominant à droite en $E_{1}$ et si $D_{2}$ est quasi dominant à droite en $E_{2}, D_{1} D_{2}$ est quasi dominant à droite en $E_{1} E_{2}$.

THÉORÈME 8. Si $E(\subset D)$ est fermé sur $D$ et dominant à droite en soi, il est majorant à droite en $D$.

DÉFINITION 4. Un point $\left(x_{0}, \vec{y}_{0}\right)$ de $D$ est dit régulier $\grave{a}$ droite par rapport à $D$ si l'on peut trouver un nombre positif $h$ tel que deux solutions, satisfaisant aux relations

$$
\vec{y}\left(x_{0}\right)=\vec{y}_{0}, \quad(x, \vec{y}(x)) \in D,
$$

coïncident dans l'intervalle $0 \leqq x-x_{0}<h$. Si l'équation (1) est régulière à droite en chaque point de $E$ par rapport à $D$, l'équation est dite régulière à droite en $E$ par rapport à $D$.

On définira de la même manière équation régulière à gauche en un point ou en un ensemble. Si une équation est régulière à droite et à gauche, elle est dite régulière.

THÉORÈME 9. Supposons remplies les hypothèses du théorème 1. Si de plus l'équation (1) est régulière à droite en $E$ par rapport à $D, E$ est majorant à droite en $D$.

En effet, soit $\vec{y}(x)$ une solution issue d'un point $\left(x_{0}, \overrightarrow{y_{0}}\right)$ de $E$, continue dans un intervalle $x_{0} \leqq x \leqq a$ et contenue dans $D$. Le théorème 1 montre qu'il existe une telle solution $\vec{y}=\vec{\varphi}(x)$ issue $\mathrm{du}$ point $\left(x_{0}, \vec{y}_{0}\right)$ et contenue dans $E$. Puisqu'il n'existe qu'une telle solution, $\vec{y}(x)$ coïncide avec $\vec{\varphi}(x)$.

3. Un lemme sur la fonction de M. KAMKE. Avant d'aller plus loin, établissons un lemme concernant la fonction de $\mathrm{M}$. KAMKE. Nous désignons toujours par $S(\vec{y})$ une fonction de M. 
KAMKE. C'est une fonction continue dans tout l'espace (à $n$ dimensions), s'annulant à l'origine et satisfaisant aux inégalités

$$
D^{ \pm} S(\vec{y}(x)) \leqq S\left(D^{ \pm} \vec{y}(x)\right)
$$

pour toute fonction $\vec{y}(x)$ admettant les dérivées à droite et à gauche. Remplaçons $\vec{y}(x)$ par $\vec{y}(-x)$ et puis $x$ par $-x$. Nous obtenons alors

$$
D^{ \pm} S(\vec{y}(x)) \geqq-S\left(-D^{ \pm} \vec{y}(x)\right) .
$$

Lemme. Si $S(\vec{y})$ est une fonction de M. Kamke, elle satisfait aux inégalités (2) et (3) pour toute fonction $\vec{y}(x)$ admettant les dérivées à droite et à gauche.

Remarquons encore qu'une fonction de M. KAMKE est positivement linéaire et convexe, et réciproquement $\left({ }^{4}\right)$.

4. Comparaison à l'aide de la fonction de M. KAMKE. Soit $\underline{\omega}(x)$ une fonction continue dans l'intervalle $a \leqq x<b$. Dans quel cas peut-on affirmer que l'ensemble $D$, défini par

$$
a \leqq x<b, S(\vec{y}) \geqq \underline{\omega}(x),
$$

est dominant à droite en un ensemble $E$ contenu dans $D$ ? Nous supposons que $\vec{f}(x, \vec{y})$ est définie dans $E$. Il est clair que $D$ est dominant à droite en un point de $E$ situé à l'intérieur, par rapport à $a \leqq x<b,|\vec{y}|<+\infty\left(^{(5)}\right.$, de $D$. Nous considérons donc un point $\left(x_{0}, \vec{y}_{0}\right)$ de $E$ situé sur la frontière, par rapport à $a \leqq x<b$, $|\vec{y}|<+\infty$, de $D$. On a évidemment

$$
a \leqq x<b, \quad S(\vec{y})=\underline{\omega}(x), \quad(x, \vec{y}) \in E
$$

pour $x=x_{0}, \vec{y}=\vec{y}_{0}$. D'après le lemme établi au $\mathrm{n}^{0}$ précédent, on a

$$
D^{+} S\left(\vec{y}_{0}+\vec{z}\left(x-x_{0}\right)\right) \geqq-S(-\vec{z}) .
$$

Supposons que $\bar{D}^{+} \underline{\omega}(x)$ prend, pour $x=x_{0}$, une valeur $\gamma_{0}$ moindre

(4) M. Hukuhara, Sur la fonction $S(x)$ de M. Kamke, Jap. Journ. Math. 17 (1941), 289-298.

(5) $|\vec{y}|$ désigne la quantité $\sqrt{y_{1}^{2}+y_{2}^{2}+\ldots+y_{n}^{2}}$. 
que $c_{0}=-S\left(-\vec{z}_{0}\right)$, où $\vec{z}_{0}=\vec{f}\left(x_{0}, \vec{y}_{0}\right)$. Si $h(>0)$ est assez petit, on aura

$$
\underline{\omega}(x) \geqq \underline{\omega}\left(x_{0}\right)+c\left(x-x_{0}\right)
$$

pour $0 \leqq x-x_{0}<h$, où $c$ est un nombre plus grand que $\gamma_{0}$ et plus petit que $c_{0}$. On choisit $\delta(>0)$ de manière que $-S(-\vec{z})>c$ pour $\left|\vec{z}-\vec{z}_{0}\right|<\dot{\delta}$. On aura alors

$$
S\left(\vec{y}_{0}+\vec{z}\left(x-x_{0}\right)\right) \geqq S\left(\vec{y}_{0}\right)+c\left(x-x_{0}\right)
$$

pour $x \geqq x_{0}$. Puisque $S\left(\vec{y}_{0}\right)=\underline{\omega}\left(x_{0}\right)$ par hypothèse, on a

$$
S\left(\vec{y}_{0}+\vec{z}\left(x-x_{0}\right)\right) \geqq \underline{\omega}(x)
$$

pour $0 \leqq x-x_{0}<h$. Ceci nous montre que le point $(x, \vec{y})$ tel que

$$
0 \leqq x-x_{0}<h,\left|\frac{\vec{y}-\vec{y}_{0}}{x-x_{0}}-\vec{f}\left(x_{0}, \overrightarrow{y_{0}}\right)\right|<\delta
$$

appartient à $D$. Nous arrivons donc au

THÉORÈmE 10. Soit $E$ un ensemble contenu dans l'ensemble $D$ défini par (4), $\underline{\omega}(x)$ étant une fonction continue dans l'intervalle $a \leqq x<b$. Si l'on a

$$
\bar{D}^{+} \underline{\omega}(x)<-S(-\vec{f}(x, \vec{y}))
$$

pour (5), $D$ est dominant à droite en $E$.

On démontre de même le

ThÉorème 11. Soit $E$ un ensemble contenu dans l'ensemble $D$ défini par

$$
a \leqq x<b, \quad S(\vec{y}) \leqq \bar{\omega}(x),
$$

$\bar{\omega}(x)$ étant une fonction continue dans l'intervalle $a \leqq x<b$. Si l'on a

$$
\underline{D}^{+} \bar{\omega}(x)>S(\vec{f}(x, \vec{y}))
$$

pour

$$
a \leqq x<b, \quad S(\vec{y})=\bar{\omega}(x), \quad(x, \vec{y}) \in E,
$$

$D$ est dominant à droite en $E$. 
Théorèmes Fondamentaux de la Théorie des Équations Dif.

En combinant les théorèmes 6,10 et 11 , nous obtenons le

ThÉORÈmE 12. Soit $E$ un ensemble contenu dans l'ensemble $D$ défini par

$$
a \leqq x<b, \quad \underline{\omega}(x) \leqq S(\vec{y}) \leqq \bar{\omega}(x),
$$

$\underline{\omega}(x)$ et $\bar{\omega}(x)$ étant des fonctions continues dans l'intervalle $a$ $\leqq x<b$. Si l'on a (6) pour (5) et (8) pour (9), $D$ est dominant à droite en $E$.

REMARQUE 1. Plaçons-nous, par exemple, dans les hypothèses du théorème 12. Si $E=D, D$ est, d'après le théorème 8 , majorant à droite en $a \leqq x<b,|\vec{y}|<+\infty$. Par suite, si une solution $\vec{y}(x)$, continue dans l'intervalle $a \leqq x<b$, satisfait aux inégalités

$$
\underline{\omega}(x) \leqq S(\vec{y}(x)) \leqq \bar{\omega}(x)
$$

pour $x=a$, ces inégalités subsistent pour $a \leqq x<b$.

Si l'on suppose au lieu de (6) et (8) les inégalités suivantes

$$
\begin{aligned}
& \bar{D}^{+} \underline{\omega}(x) \leqq-S(-\vec{f}(x, \vec{y})), \\
& \underline{D}^{+} \bar{\omega}(x) \geqq S(\vec{f}(x, \vec{y})),
\end{aligned}
$$

peut-on dire que $D$ est quasi dominant à droite? La réponse est affirmative.

THÉorÈme 13. Soit $E$ un ensemble contenu dans l'ensemble $D$ défini par (10), $\underline{\omega}(x)$ et $\bar{\omega}(x)$ étant des fonctions continues dans l'intervalle $a \leqq x<b$. Si $S(\vec{y})$ ne prend pas de valeurs négatives, nous supposons de plus

$$
\bar{\omega}(x) \geqq 0, \quad \underline{D}^{+} \bar{\omega}(x) \geqq 0, \quad \bar{D}^{+} \underline{\omega}(x) \leqq 0 .
$$

Si l'on a (11) pour (5) et (12) pour (9), $D$ est quasi dominant, à droite en $E$.

Soit $\left(x_{0}, \vec{y}_{0}\right)$ un point de $E$ situé sur la frontière, par rapport à $a \leqq x<b,|\vec{y}|<+\infty$, de $D$. Il suffit de montrer que $D$ est quasi dominant à droite en $\left(x_{0}, \vec{y}_{0}\right)$. On aura (5) ou (9) pour $x=x_{0}, \vec{y}=\vec{y}_{0}$. Supposons, par exemple, que l'on a (5) pour $x=x_{0}$, $\vec{y}=\vec{y}_{0}$. Posons 


$$
\vec{z}_{0}=\vec{f}\left(x_{0}, \vec{y}_{0}\right), \quad c_{0}=-S\left(-\vec{z}_{0}\right), \quad c_{0}^{\prime}=S\left(\vec{z}_{0}\right)
$$

Puisque

$$
S\left(\vec{z}_{0}\right)+S\left(-\vec{z}_{0}\right) \geqq S(\overrightarrow{0})=0
$$

on a $c_{0} \leqq c_{0}^{\prime}$. Soit $c$ la valeur de $D^{+} S\left(\vec{y}_{0}+x \vec{z}_{0}\right)$ pour $x=0$. Les inégalités (2) et (3) montrent que l'on a $c_{0} \leqq c \leqq c_{0}^{\prime}$. Nous prenons une fonction $\omega(x)$, continue dans l'intervalle $x_{0} \leqq x<b$ et satisfaisant aux relations

$$
\underline{\omega}(x) \leqq \omega(x) \leqq \bar{\omega}(x)
$$

pour $x_{0} \leqq x<b$ et

$$
\omega(x)=\underline{\omega}(x), \quad D^{+} \omega(x)=c
$$

pour $x=x_{0}$. Si $c>0$, on aura $\omega(x)>\omega\left(x_{0}\right)$ pour $0<x-x_{0}<h$, $h$ désignant un nombre positif assez petit. La fonction

$$
\chi(t)=S\left(\overrightarrow{y_{0}}+t \overrightarrow{z_{0}}\right)
$$

étant convexe et admettant la dérivée à droite positive pour $t=0$, elle est croissante pour $t>0$. On peut donc déterminer la fonction $\lambda(x)(\geqq 0)$ par la relation

$$
S\left(\vec{y}_{0}+\lambda(x) \vec{z}_{0}\right)=\omega(x)
$$

pour $0 \leqq x-x_{0}<h$. Cette fonction est continue et positive pour $0<x-x_{0}<h$ et s'annule pour $x=x_{0}$. En faisant $x \rightarrow x_{0}+0$ dans la relation

$$
\frac{\omega(x)-\omega\left(x_{0}\right)}{x-x_{0}}=\frac{\chi(\lambda(x))-\chi\left(\lambda\left(x_{0}\right)\right)}{\lambda(x)-\lambda\left(x_{0}\right)} \cdot \frac{\lambda(x)-\lambda\left(x_{0}\right)}{x-x_{0}},
$$

on voit

$$
\lim _{x \rightarrow x_{0}+0} \frac{\lambda(x)}{x-x_{0}}=1
$$

La courbe $\vec{y}=\vec{y}_{0}+\lambda(x) \vec{z}_{0}, 0 \leqq x-x_{0}<h$ est contenue dans $D$ et $D^{+}\left(\vec{y}_{0}+\lambda(x) \vec{z}_{0}\right)$ prend la valeur $\vec{z}_{0}$ pour $x=x_{0}$. $D$ est donc quasi dominant à droite en $\left(x_{0}, \vec{y}_{0}\right)$. Il en sera de même si $c<0$. 
Si $c=0$, nous distinguons deux cas suivant que $S(\vec{y})$ prend des valeurs négatives ou non.

Le cas oì $S(\vec{y})$ prend des valeurs négatives. Soit $\vec{u}_{0}$ un point où $S(\vec{y})$ prend la valeur -1 . Puisque la somme de $S\left(\vec{u}_{0}\right)$ et de $S\left(-\vec{u}_{0}\right)$ est non négative, la valeur $S\left(-\vec{u}_{0}\right)$ est au moins égale à 1. Nous prenons comme plus haut une fonction $\omega(x)$ jouissant des propriétés analogues. La fonction de $t: S\left(\vec{y}+t \vec{u}_{0}\right)$ décroît de $+\infty$ à $-\infty$ lorsque $t$ varie de $-\infty$ à $+\infty$. La relation

$$
S\left(\vec{y}_{0}+\left(x-x_{0}\right) \vec{z}_{0}+\lambda(x) \vec{u}_{0}\right)=\omega(x)
$$

définit donc une fonction $\lambda(x)$ continue dans l'intervalle $x_{0} \leqq x<b$. Il suffit de montrer que l'on a

$$
\lim _{x \rightarrow x_{0}+0} \frac{\lambda(x)}{x-x_{0}}=0 .
$$

Car alors la courbe

$$
\vec{y}=\vec{y}_{0}+\left(x-x_{0}\right) \vec{z}_{0}+\lambda(x) \vec{u}_{0}, \quad x_{0} \leqq x<b
$$

est contenue dans $D$, et la dérivée à droite de

$$
\vec{y}_{0}+\left(x-x_{0}\right) \vec{z}_{0}+\lambda(x) \vec{u}_{0}
$$

prend la valeur $\vec{z}_{0}$ pour $x=x_{0}$.

Si $\lambda(x)>0$, on a

$$
\begin{aligned}
\omega(x)-\omega\left(x_{0}\right) & \leqq S\left(\vec{y}_{0}+\left(x-x_{0}\right) \vec{z}_{0}\right)+S\left(\lambda(x) \vec{u}_{0}\right)-S\left(\vec{y}_{0}\right) \\
& =S\left(\vec{y}_{0}+\left(x-x_{0}\right) \vec{z}_{0}\right)-S\left(\vec{y}_{0}\right)-\lambda(x) .
\end{aligned}
$$

En divisant l'inégalité ainsi obtenue par $x-x_{0}$ et faisant $x \rightarrow x_{0}+0$, nous obtenons

$$
\varlimsup_{x \rightarrow x_{0}+0} \frac{\lambda(x)}{x-x_{0}} \leqq 0
$$

Si $\lambda(x)<0$, on a

$$
\begin{aligned}
\omega(x)-\omega\left(x_{0}\right) & \geqq S\left(\vec{y}_{0}+\left(x-x_{0}\right) \vec{z}_{0}\right)-S\left(-\lambda(x) \vec{u}_{0}\right)-S\left(\vec{y}_{0}\right) \\
& =S\left(\vec{y}_{0}+\left(x-x_{0}\right) \vec{z}_{0}\right)-S\left(\vec{y}_{0}\right)-\lambda(x) .
\end{aligned}
$$


En divisant l'inégalité ainsi obtenue par $x-x_{0}$ et faisant $x \rightarrow x_{0}+0$, nous obtenons

$$
\lim _{x \rightarrow x_{0}+0} \frac{\lambda(x)}{x-x_{0}} \geqq 0 .
$$

Le cas où $S(\vec{y})$ ne prend pas de valeurs négatives. If suffit de montrer que l'on a

$$
\underline{\omega}(x) \leqq S\left(\vec{y}_{0}+\left(x-x_{0}\right) \vec{z}_{0}\right) \leqq \bar{\omega}(x)
$$

dans un intervalle assez petit $0 \leqq x-x_{0}<h$. Si $c_{0}<0$, on a $c_{0}^{\prime} \geqq-c_{0}>0$ et ces inégalités sont évidentes. Si $c_{0}=0$, on a

$$
S\left(\vec{y}_{0}+\left(x-x_{0}\right) \vec{z}_{0}\right) \geqq S\left(\vec{y}_{0}\right)-\left(x-x_{0}\right) S\left(-\vec{z}_{0}\right)=S\left(\vec{y}_{0}\right) \geqq \underline{\omega}(x)
$$

pour $x_{0} \leqq x<b$. Si $c_{0}^{\prime}>0$, la deuxième des inégalités (14) est évidente. Si $c_{0}^{\prime}=0$, on a

$$
S\left(\vec{y}_{0}+\left(x-x_{0}\right) \vec{z}_{0}\right) \leqq S\left(\vec{y}_{0}\right)+\left(x-x_{0}\right) S\left(\vec{z}_{0}\right)=S\left(\vec{y}_{0}\right) \leqq \bar{\omega}(x)
$$

pour $x_{0} \leqq x<b$. Les inégalités (14) sont donc démontrées.

Si $E=D, D$ est quasi dominant à droite en soi. L'ensemble défini par $a \leqq x<b,|\vec{y}|<+\infty$ est évidemment dominant à droite en $D$. Nous arrivons donc, en appliquant le théorème 1, $\mathrm{au}$

Corollaire. Plaçons-nous dans les hypothèses du théorème. Supposons de plus que $E=D$ et que $\vec{f}(x, \vec{y})$ est continue dans $D$. $\mathrm{Si}$

$$
\underline{\omega}(a) \leqq S(\vec{\beta}) \leqq \bar{\omega}(a),
$$

il existe au moins une solution $\vec{y}(x)$ continue dans un certain intervalle $a \leqq x<c$, satisfaisant aux inégalités

$$
\underline{\omega}(x) \leqq S(\vec{y}(x)) \leqq \bar{\omega}(x),
$$

et prenant la valeur $\vec{\beta}$ pour $x=a$. Si l'on ne peut prolonger cette solution au delà de $x=c,|\vec{y}(x)|$ croît indéfiniment lorsque $x \rightarrow c-0$.

Remarque 2. Si $S(\vec{y})$ est positive sauf pour $\vec{y}=\overrightarrow{0},|\vec{y}(x)|$ ne peut croître indéfiniment lorsque $x \rightarrow c-0$, car l'ensemble défini 
par $S(\vec{y}) \leqq M$ est borné. Par suite la solution $\vec{y}(x)$ du corollaire peut être supposée continue pour $a \leqq x<b$.

REMARQUE 3. On peut remplacer, dans ces théorèmes 10-13, l'intervalle $a \leqq x<b$ par l'intervalle ouvert $a<x<b$.

THÉORÈme 14. Soient $S(\vec{y})$ úne fonction de M. KAMKE positive sauf pour $\vec{y}=\overrightarrow{0}$, $\underline{\omega}(x)$ et $\bar{\omega}(x)$ des fonctions continues dans l'intervalle $a<x<b$ et telles que

$$
\underline{\omega}(x) \leqq \bar{\omega}(x), \quad \bar{\omega}(x) \geqq 0,
$$

et $\vec{f}(x, \vec{y})$ une fonction continue dans l'ensemble défini par

$$
a<x<b, \underline{\omega}(x) \leqq S(\vec{y}) \leqq \bar{\omega}(x) .
$$

Supposons de plus que l'on a (11) pour

$$
a<x<b, S(\vec{y})=\underline{\omega}(x),
$$

et (12) pour

$$
a<x<b, \quad S(\vec{y})=\bar{\omega}(x) .
$$

Il existe alors au moins une solution continue dans l'intervalle $a<x<b$ et contenue dans $D$.

$\bar{\omega}(x)$ est nécessairement non décroissante. On peut supposer que $\omega(x)$ est non croissante, sinon, on la remplacerait par $\max \{\underline{\omega}(x), 0\}$. Les relations (13) sont alors remplies. Prenons une suite décroissante $\left\{a_{m}\right\}$ convergeant vers $a$. D'après la remarque 2 , il existe une solution $\vec{y}=\vec{\varphi}_{m}(x)$ continue dans l'intervalle $a_{m} \leqq x$ $<b$. On peut extraire de la suite $\left\{\vec{\varphi}_{m}(x)\right\}$ une suite partielle convergente dans l'intervalle $a<x<b$. La limite de cette suite partielle répond à la question.

Nous désignerons toujours par $\mathfrak{F}$ une famille de fonctions jouissant des propriétés suivantes:

(a) Une fonction de $\mathfrak{F}$ est continue dans un intervalle ouvert, dont l'extrémité gauche est $a$.

(b) Si deux fonctions $f(x)$ et $g(x)$, appartenant à $\mathfrak{F}$, satisfont à l'inégalité $f(x) \leqq g(x)$ dans un certain intervalle, dont l'extrémité gauche est $a$, toute fonction continue $h(x)$, qui satisfait aux inégalités $f(x) \leqq h(x) \leqq g(x)$ au moins dans un intervalle assez petit $0<x-a<\delta$, appartient à $\mathfrak{F}$. 
THÉORÈME 15. Supposons que la famille $\mathfrak{F}$ contient la fonction identiquement nulle et que la fonction $S(\vec{y})$ est positive sauf pour $\vec{y}=\overrightarrow{0}$. Soient $\bar{\omega}(x)$ une fonction continue et non négative dans l'intervalle $a<x<b$ et appartenant à $\mathfrak{乛}$ et $f(x, y)$ une fonction continue dans un ensemble $D$ qui contient l'ensemble $E$ défini par

$$
a<x<b, \quad S(\vec{y}) \leqq \bar{\omega}(x)
$$

et telle que l'on a (12) pour (17). Si l'équation n'admet qu'une solution $\vec{y}(x)$ continue dans l'intervalle $a<x<b$ et telle que $S(\vec{y}(x))$ appartient à $\mathfrak{F}$, cette solution satisfait à l'inégalité $S(\vec{y}(x)) \leqq \vec{\omega}(x)$ dans l'intervalle $a<x<b$.

Car le théorème 14 montre qu'il existe une solution $\vec{y}=\vec{\varphi}(x)$ satisfaisant à l'inégalité $S(\vec{\varphi}(x)) \leqq \bar{\omega}(x)$ pour $a<x<b\left({ }^{6}\right) . \quad S(\vec{\varphi}(x))$ appartient à la famille $\widetilde{F}$. Une telle solution étant unique, on a $\vec{y}(x)=\vec{\varphi}(x)$.

5. Comparaison d'un système différentiel avec une seule équation différentielle. Considérons le cas où $\underline{\omega}(x)$ est une solution de l'équation

$$
Y^{\prime}=\underline{F}(x, Y) .
$$

Les inégalités (6) et (11) sont respectivement les conséquences de

$$
\underline{F}(x, S(\vec{y}))<-S(-\vec{f}(x, \vec{y}))
$$

et

$$
\underline{F}(x, S(\vec{y})) \leqq-S(-\vec{f}(x, \vec{y})) .
$$

Si $\bar{\omega}(x)$ est une solution de l'équation

$$
Y^{\prime}=\bar{F}(x, Y) \text {, }
$$

les inégalités

$$
\bar{F}(x, S(\vec{y}))>S(\vec{f}(x, \vec{y}))
$$

et

$$
\bar{F}(x, S(\vec{y})) \geqq S(\vec{f}(x, \vec{y}))
$$

(6) On prend, par exemple, $\omega(x)=0$. 
entraînen t respectivement (8) et (12). Par suite, dans le cas où $\underline{\omega}(x)$ est une solution de (19), continue dans l'intervalle $a \leqq x<b$, le théorème 10, par exemple, peut s'énoncer comme il suit:

Si l'on a $\left(6^{\prime}\right)$ pour (5), l'ensemble défini par (4) est dominant à droite en $E$.

On peut faire des remarques analogues concernant les théorèmes 11-15.

On rencontre le plus souvent, dans les applications, le cas où l'équation auxiliaire est régulière à droite. On peut alors utiliser les deux théorèmes suivants.

THÉORÈmE 16. Soient $\underline{\omega}(x)$ et $\bar{\omega}(x)$ des fonctions continues dans l'intervalle $a \leqq x<b$ et telles que $\underline{\omega}(x)<\bar{\omega}(x)$, et $\underline{F}(x, Y)$ une fonction continue dans l'ensemble $\Delta$ défini par

$$
a \leqq x<b, \quad \underline{\omega}(x)<Y \leqq \bar{\omega}(x) .
$$

Supposons que l'équation (19) est régulière à droite en $\Delta$ par rapport à $\Delta$ lui-même et admet une solution $\omega(x)$ continue dans l'intervalle $a \leqq x<b$ et contenue dans $\Delta$ et que l'on a $\left(11^{\prime}\right)$. pour

$$
a \leqq x<b, \quad \underline{\omega}(x)<S(\vec{y}) \leqq \bar{\omega}(x), \quad(x, \vec{y}) \in E,
$$

$E$ désignant l'ensemble où $\vec{f}(x, \vec{y})$ est définie. Alors l'ensemble $D$ défini par

$$
a \leqq x<b, \quad S(\vec{y}) \geqq \omega(x)
$$

est majorant à droite en $a \leqq x<b, \quad|\vec{y}|<+\infty$.

Soit, en effet, $\vec{y}(x)$ une solution de (1), continue dans un intervalle $x_{0} \leqq x<c \quad\left(a \leqq x_{0}<c \leqq b\right)$ et satisfaisant à l'inégalité $\left.\overrightarrow{S(y}\left(x_{0}\right)\right) \geqq \omega\left(x_{0}\right)$. Il suffit de montrer que l'on a $S(\vec{y}(x)) \geqq \omega(x)$ pour $x_{0} \leqq x<c$. Si l'on n'avait pas cette inégalité, on pourrait trouver deux nombres $x_{1}$ et $x_{2}\left(x_{0} \leqq x_{1}<x_{2} \leqq c\right)$ tels que l'on a

$$
\underline{\omega}(x)<S(\vec{y}(x))<\omega(x)
$$

pour $x_{1}<x<x_{2}$ et $S\left(\vec{y}\left(x_{1}\right)\right)=\omega\left(x_{1}\right)$. Puisque l'on a

$$
D^{+} S(\vec{y}(x)) \geqq-S(-\vec{f}(x, \vec{y}(x)) \geqq \underline{F}(x, S(\vec{y}(x))),
$$

la fonction $S(\vec{y}(x))$ serait, dans l'intervalle, quasi supérieure à droite pour (19). Elle serait donc, d'après l'analogue du théorème 
14 du Premier Mémoire, majorante à droite pour (19). Puisque $\omega(x)$ est une solution de (19) prenant la même valeur que $S(\vec{y}(x))$ pour $x=x_{1}$, on aurait $S(\vec{y}(x)) \geqq \omega(x)$ pour $x_{1} \leqq x<x_{2}$, ce qui est contraire à l'hypothèse.

On démỏntre de même le

THÉORÈmE 17. Soient $\underline{\omega}(x)$ et $\bar{\omega}(x)$ des fonctions continues dans l'intervalle $a \leqq x<b$ et telles que $\underline{\omega}(x)<\bar{\omega}(x)$, et $\bar{F}(x, Y)$ une fonction continue dans l'ensemble $\Delta$ défini par

$$
a \leqq x<b, \quad \underline{\omega}(x) \leqq Y<\bar{\omega}(x) .
$$

Supposons que l'équation $\left(19^{\prime}\right)$ est régulière à droite en $\Delta$ par rapport à $\Delta$ lui-même et admet une solution $\omega(x)$ continue dans l'intervalle $a \leqq x<b$ et contenue dans $\Delta$ et que l'on a (12') pour

$$
a \leqq x \leqslant b, \quad \underline{\omega}(x) \leqq S(\vec{y})<\bar{\omega}(x), \quad(x, \vec{y}) \in E,
$$

$E$ désignant l'ensemble où $\vec{f}(x, \vec{y})$ est définie. Alors l'ensemble $D$ défini par

$$
a \leqq x<b, \quad S(\vec{y}) \leqq \omega(x),
$$

est majorant à droite en $a \leqq x<b,|\vec{y}|<+\infty$.

REMARQUE. On peut remplacer, dans ces deux théorèmes, l'intervalle $a \leqq x<b$ par l'intervalle ouvert $a<x<b$.

THÉORÈmE 18. Soient $\underline{\omega}(x)$ et $\vec{\omega}(x)$ des fonctions continues dans l'intervalle $a<x<b$ et telles que $\underline{\omega}(x)<\bar{\omega}(x)$, et $\underline{F}(x, Y)$ une fonction continue dans l'ensemble $\Delta$ défini par (20). Supposons que l'on a $\left(11^{\prime}\right)$ pour (21), $E$ désignant l'ensemble où $\vec{f}(x, \vec{y})$ est définie, et que l'équation (19) admet une solution $Y=\omega(x)$ et une seule appartenant à la famille $\mathfrak{F}$, et contenue dans $\Delta$, cette solution étant continue dans l'intervalle $a<x<b$. S'il existe une solution $\vec{y}=\vec{\varphi}(x)$ de (1), continue dans l'intervalle $a<x<b$, satisfaisant à l'inégalité $\underline{\omega}(x)<S(\vec{\varphi}(x))$ dans un intervalle assez petit $0<x-a<h$ et telle que $S(\vec{\varphi}(x))$ appartient à $\mathfrak{F}$, on a l'inégalité $S(\vec{\varphi}(x)) \geqq \omega(x)$ pour $a<x<b$.

En effet, on voit sans peine que $S(\vec{\varphi}(x))$ est quasi supérieure à droite pour (19). L'analogue du théorème $15 \mathrm{du}$ Premier Mémoire, 
amélioré à la fin de notre présent mémoire, montre que l'on a $S(\vec{\varphi}(x)) \geqq \omega(x)$ tant que l'on a $S(\vec{\varphi}(x))>\underline{\omega}(x)$.

Si l'on a $S \cdot(\vec{\varphi}(x)) \geqq \omega(x)$ dans un intervalle $a<x<c(<b)$, l'inégalité $S(\vec{\varphi}(x))>\underline{\omega}(x)$ subsiste au delà de $x=c$. Puisqu'on a celle-ci dans un intervalle assez petit $0<x-a<h$ par hypothèse, celle-là subsiste dans l'intervalle $a<x<b$.

On démontre de même le

THÉORÈME 19. Soient $\underline{\omega}(x)$ et $\bar{\omega}(x)$ des fonctions continues dans l'intervalle $a<x<b$ et telles que $\omega(x)<\bar{\omega}(x)$, et $\bar{F}(x, Y)$ une fonction continue dans l'ensemble $\Delta$ défini par (22). Supposons que l'on a (12') pour (23), $E$ désignant l'ensemble où $\vec{f}(x, \vec{y})$ est définie, et que l'équation $\left(19^{\prime}\right)$ admet une solution $Y=\omega(x)$ et une seule appartenant à la famille $\mathfrak{F}$ et contenue dans $\Delta$, cette solution étant continue dans l'intervalle $a<x<b$. S'il existe une solution de (1) continue dans l'intervalle $a<x<b$, satisfaisant à l'inégalité $S(\vec{\varphi}(x))<\omega(x)$ dans un intervalle assez petit $0<x-a<h$ et telle que $S(\vec{\varphi}(x))$ appartient à $\mathfrak{F}$, on a l'inégalité $S(\vec{\varphi}(x)) \leqq \omega(x)$ pour $a<x<b$.

6. Théorème d'unicité. Le théorème 19 nous conduit immédiatẹment au théorème d'unicité suivant.

THÉORÈME 20. Soient $\omega(x)$ une fonction continue et positive dans l'intervalle $a<x<b, \bar{F}(x, Y)$ une fonction continue dans l'ensemble $\Delta$ défini par

$$
a<x<b, \quad 0 \leqq Y<\omega(x),
$$

et $\vec{f}(x, \vec{y})$ une fonction définie dans un certain ensemble $D$ et satisfaisant à l'inégalité

$$
S\left(\vec{f}\left(x, \overrightarrow{y^{1}}\right)-\vec{f}\left(x, \overrightarrow{y^{2}}\right)\right) \leqq \bar{F}\left(x, S\left(\vec{y}^{1}-\vec{y}^{2}\right)\right)
$$

pour

$$
a<x<b, \quad\left(x, \overrightarrow{y^{1}}\right) \in D, \quad\left(x, \overrightarrow{y^{2}}\right) \in D, \quad S\left(\overrightarrow{y^{1}}-\vec{y}^{2}\right)<\omega(x),
$$

$S(\vec{y})$ étant positive sauf pour $\vec{y}=\overrightarrow{0}$. Supposons de plus que la famille $\mathfrak{\Im}$ contient la fonction identiquement nulle, que toute fonction $f(x)$ appartenant à la famile $\mathfrak{F}$ satisfait à l'inégalité $f(x)<\omega(x)$ dans un intervalle assez petit, dont l'extrémité gauche est $a$, et 
que la solution de l'équation $\left(19^{\prime}\right)$ appartenant à $\mathfrak{F}$ est unique et identiquement nulle. Alors deux solutions $\vec{\varphi}^{1}(x)$ et $\vec{\varphi}^{2}(x)$ de l'équation (1), continues pour $a<x<b$ et telles que $S\left(\vec{\varphi}^{1}(x)-\vec{\varphi}^{2}(x)\right)$ appartient à $\mathfrak{F}$, coïncident identiquement dans l'intervalle $a<x<b$.

Soit, en effet,

$$
\overrightarrow{z^{\prime}}=\vec{g}(x, \vec{z})
$$

l'équation en $\vec{z}=\vec{y}-\vec{\varphi}^{2}(x)$. La fonction

satisfait à l'inégalité

$$
\vec{g}(x, \vec{z})=\vec{f}\left(x, \vec{z}+\vec{\varphi}^{2}(x)\right)-\vec{f}\left(x, \vec{\varphi}^{2}(x)\right)
$$

$$
S(\vec{g}(x, \vec{z})) \leqq \bar{F}(x, S(\vec{z}))
$$

pour

$$
a<x<b, \quad\left(x, \vec{z}+\vec{\varphi}^{2}(x)\right) \in D, \quad S(\vec{z})<\omega(x) .
$$

Cette équation en $\vec{z}$ admet une solution $\vec{z}=\vec{\varphi}^{1}(x)-\vec{\varphi}^{2}(x)$ continue dans l'intervalle $a<x<b$, et telle que $S(\vec{z})$ appartient à $\mathfrak{\mho}$. Le théorème 19 montre donc que l'on a

$$
S\left(\dot{\varphi}^{1}(x)-\vec{\varphi}^{2}(x)\right) \leqq 0
$$

ce qui entraîne $\vec{\varphi}^{1}(x)=\vec{\varphi}^{2}(x)$.

7. Continuité. Nous allons considérer le système différentiel contenant des paramètres. Nous désignons par une lettre en caractère gras un point dans l'espace à $m$ dimensions de sorte que

$$
\overrightarrow{y^{\prime}}=\vec{f}(x, \vec{y}, \mathbf{t})
$$

représente un système de $n$ équations différentielles contenant $m$ paramètres $t_{1}, t_{2}, \ldots, t_{m}$. On démontre sans peine le

THÉORÈME 21. Soit $\vec{f}(x, \vec{y}, \mathbf{t})$ une fonction continue par rapport à $(x, \vec{y}, \mathbf{t})$ pour

$$
a<x<b, \quad S(\vec{y}) \leqq \omega(x), \quad \mathbf{t} \in T,
$$

$\omega(x)$ étant continue et non négative pour $a<x<b, \mathrm{~S}(\vec{y})$ positive sauf pour $\vec{y}=\overrightarrow{0}$ et $T$ un ensemble dans l'espace à $m$ dimensions. Supposons que l'équation (24) admet pour chaque valeur de $\mathbf{t}(\epsilon T)$ 
Théorèmes Fondamentaux de la Théorie des Équations Dif.

une solution et une seule $\vec{y}=\vec{\varphi}(x, \mathbf{t})$ telle que $S(\vec{y}) \leqq \omega(x)$. Alors $\vec{\varphi}(x, \mathbf{t})$ est continue par rapport à $(x, \mathbf{t})$ pour

$$
a<x<b, \quad \mathrm{t} \in T \text {. }
$$

REMARQUE. Nous avons supposé que les variables sont réelles. Mais il est seulement essentiel que la variable $x$ est réelle. On peut donc supposer que, dans les théorèmes établis jusqu'ici, les variables $y_{1}, \ldots, y_{n}$ ainsi que $t_{1}, \ldots, t_{m}$ sont complexes.

8. Dérivabilité. Démontrons maintenant le théorème de dérivabilité suivant, l'objet principal de ce mémoire.

THÉORÈmE 22. Nous supposons remplies les hypothèses suivantes:

$1^{\circ} \quad \overrightarrow{(\vec{y})}$ est positive sauf pour $\vec{y}=\overrightarrow{0}$.

$\left.2^{\circ} \quad \omega(x) \geqq 0\right), p(x), q(x)$ sont continues pour $a<x<b$.

$3^{\circ}$ La famille $\tilde{f}$ contient la fonction identiquement nulle.

$4^{\circ}$ Les fonctions

$$
\begin{aligned}
& \vec{f}(x, \vec{y}, \lambda), \vec{f}_{\lambda}^{\prime}(x, \vec{y}, \lambda), \\
& \frac{\partial}{\partial y_{j}} \vec{f}(x, \vec{y}, \lambda) \quad(j=1,2, \ldots, n)
\end{aligned}
$$

sont continues par rapport à $(x, \vec{y}, \lambda)$ pour

$$
a<x<b, \quad S(\vec{y}) \leqq \omega(x), \quad \underline{\lambda} \leqq \lambda \leqq \bar{\lambda},
$$

et satisfont à l'inégalité

$$
S\left(\sum_{k=1}^{n} \frac{\partial \vec{f}}{\partial y_{k}} z_{k}+\frac{\partial \vec{f}}{\partial \dot{\lambda}}\right) \leqq p(x) S(\vec{z})+q(x) .
$$

$5^{\circ} \quad$ L'équation

$$
\overrightarrow{y^{\prime}}=\vec{f}(x, \vec{y}, \lambda)
$$

admet, pour chaque valeur de $\lambda$ dans l'intervalle $\underline{\lambda} \leqq \lambda \leqq \bar{\lambda}$, une solution $\vec{y}=\vec{\varphi}(x, \lambda)$ satisfaisant à l'inégalité

$$
S(\vec{\varphi}(x, \lambda)) \leqq \omega(x)
$$

dans l'intervalle $a<x<b$ et telle que 


$$
S\left(\frac{\vec{\varphi}\left(x, \lambda_{1}\right)-\vec{\varphi}\left(x, \lambda_{2}\right)}{\lambda_{1}-\lambda_{2}}\right)
$$

appartient à $\mathfrak{F}, \lambda_{1}$ et $\lambda_{2}$ désignant des nombres quelconques dans l'intervalle $\underline{\lambda} \leqq \lambda \leqq \bar{\lambda}$.

$6^{\circ} \quad$ L'équation

$$
Y^{\prime}=p(x) Y+q(x)
$$

admet une solution $Y=\eta(x)$ et une seule appartenant à $\mathfrak{F}$.

Dans ces hypothèses $\vec{\varphi}(x, \lambda)$ est continue par rapport à $(x, \lambda)$, ainsi que sa dérivée $\vec{\varphi}_{\lambda}^{\prime}(x, \lambda)$, pour $a<x<b, \quad \underline{\lambda} \leqq \lambda \leqq \bar{\lambda}$.

Soit $\lambda_{0}$ une valeur quelconque dans l'intervalle $\underline{\lambda} \leqq \lambda \leqq \vec{\lambda}$. Soit

$$
\overrightarrow{z^{\prime}}=\vec{g}(x, \vec{z}, \lambda)
$$

l'équation en

$$
\vec{y}=\vec{\varphi}\left(x, \lambda_{0}\right)+\left(\lambda-\lambda_{0}\right) \vec{z} .
$$

Le second membre est donné par

$$
\vec{g}(x, \vec{z}, \lambda)=\frac{1}{\lambda-\lambda_{0}}\left\{\vec{f}(x, \vec{y}, \vec{\lambda})-\vec{f}\left(x, \vec{\varphi}^{0}, \lambda_{0}\right)\right\},
$$

où $\vec{\varphi}^{0}=\vec{\varphi}\left(x, \lambda_{0}\right)$. Cette équation admet une solution appartenant à $\mathfrak{F}:$

$$
z=\frac{1}{\lambda-\lambda_{0}}\left\{\vec{\varphi}(x, \lambda)-\vec{\varphi}\left(x, \lambda_{0}\right)\right\} .
$$

L’inégalité (26) entraîne

$$
S(\vec{g}(x, \vec{z}, \lambda)) \leqq p(x) S(\vec{z})+q(x)
$$

pour (25), où l'on doit remplacer $\vec{y}$ par le second membre de (30). En posant $\stackrel{\vec{z}}{z}=\overrightarrow{0}$ dans (26), nous obtenons $q(x) \geqq 0$. Le théorème $20 \mathrm{du}$ Premier Mémoire montre que $\eta(x) \geqq 0$. Le théorème. 19 est donc applicable et l'on a

$$
S\left(\frac{\vec{\varphi}(x, \lambda)-\vec{\varphi}\left(x, \lambda_{0}\right)}{\lambda-\lambda_{0}}\right) \leqq \eta(x),
$$

d'où il résulte la continuité de $\vec{\varphi}(x, \lambda)$ par rapport à $(x, \lambda)$. 
$\rightarrow$ La dérivée $\vec{\varphi}_{\lambda}^{\prime}(x, \lambda)$, s'il existe, doit satisfaire à l'équation en $u$ :

$$
\overrightarrow{u^{\prime}}=\sum_{k=1}^{n} \vec{h}_{k}(x, \lambda) u_{k}+\vec{h}(x, \lambda)
$$

où $\vec{h}_{k}(x, \lambda)$ et $\vec{h}(x, \lambda)$ représentent les fonctions que l'on obtient en remplaçant $\vec{y}$ par $\vec{\varphi}(x, \lambda)$ dans $\frac{\partial \vec{f}}{\partial y_{k}}$ et $\frac{\partial \vec{f}}{\partial \lambda}$. Elles sont donc continues par rapport à $(x, \lambda)$ pour $a<x<b, \underline{\lambda} \leqq \lambda \leqq \bar{\lambda}$. Puisqu'on a, d'après (26),

$$
S\left(\sum_{k=1}^{n} \vec{h}_{k}(x, \lambda) u_{k}+\vec{h}(x, \lambda)\right) \leqq p(x) S(\vec{u})+q(x),
$$

l'ensemble

$$
a<x<b, \quad S(\vec{u}) \leqq \eta(x)
$$

est quasi dominant à droite. Par suite l'équation (33) admet au moins une solution continue (par rapport à $x$ ) pour $a<x<b$ $(\underline{\lambda} \leqq \lambda \leqq \bar{\lambda})$ et satisfaisant à l'inégalité $S(\vec{u}) \leqq \eta(x)$. S'il existe deux telles solutions, leur différence $\vec{v}$ satisfait à la relation

$$
S(\vec{v}) \leqq(1+K) \eta(x)=o\left(e^{P(x)}\right),
$$

où

$$
K=\max \{S(-\vec{y}) / S(\vec{y})\}, \quad P(x)=\int p(x) d x .
$$

En posant $\vec{u}=M \vec{v}$ dans (34) et en divisant par $M(<0)$, nous obtenons

$$
S\left(\sum_{k=1}^{n} \vec{h}_{k}(x, \lambda) v_{k}+\frac{1}{M} \vec{h}(x, \lambda)\right) \leqq p(x) S(\vec{v})+\frac{1}{M} q(x) .
$$

En y faisant $M \rightarrow+\infty$, on obtient

$$
S\left(\sum_{k=1}^{n} h_{k}(x, \lambda) v_{k}\right) \leqq p(x) S(\vec{v})
$$

La solution, de l'équation $Y^{\prime}=p(x) Y$, telle que $Y=o\left(e^{P(x)}\right)$ étant unique et identiquement nulle, le théorème 20 montre, en prenant pour $\mathfrak{F}$ la famille de fonctions $o\left(e^{P(x)}\right)$, que $\vec{v}$ doit être identique- 
ment nulle. La solution, de (33), telle que $S(\vec{u}) \leqq \eta(x)$ est donc unique. Nous la désignons par $\vec{u}=\vec{\psi}(x, \lambda)$. Nous obtenons

$$
S(\vec{\psi}(x, \lambda)) \leqq \eta(x)
$$

pour $a<x<b, \underline{\lambda} \leqq \lambda \leqq \bar{\lambda}$. Nous allons montrer que $\vec{\psi}(x, \lambda)$ est la dérivée de $\vec{\varphi}(x, \lambda)$ par rapport à $\lambda$.

Pour cela, prenons une valeur quelconque $\lambda_{0}$ dans l'intervalle $\underline{\lambda} \leqq \lambda \leqq \bar{\lambda}$ et posons

$$
\begin{aligned}
& \vec{\varphi}^{0}(x)=\vec{\varphi}\left(x, \lambda_{0}\right), \quad \vec{\phi}^{0}(x)=\overrightarrow{\phi^{0}}\left(x, \lambda_{0}\right), \\
& \vec{\varphi}(x, \lambda)=\vec{\varphi}^{0}(x)+\left(\lambda-\lambda_{0}\right)\left\{\vec{\psi}^{0}(x)+\vec{\chi}(x, \lambda)\right\} .
\end{aligned}
$$

Il suffit de démontrer la relation

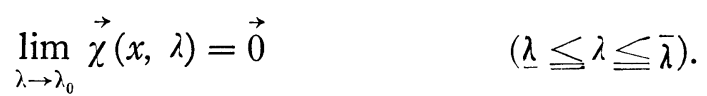

L'inégalité (32) entraîne

$$
S\left(\vec{\psi}^{0}(x)+\vec{\chi}(x, \lambda)\right) \leqq \eta(x) .
$$

On en déduit, en tenant compte de (35),

$$
S \overrightarrow{(\chi}(x, \lambda)) \leqq(1+K) \eta(x) .
$$

$\vec{v}=\vec{\chi}(x, \lambda)$ satisfait à l'équation

$$
\overrightarrow{v^{\prime}}=\frac{\vec{f}-\vec{f}^{0}}{\lambda-\lambda_{0}}-\left(\sum_{k=1}^{n} \frac{\partial \vec{f}^{0}}{\partial \varphi_{k}^{0}} \psi_{k}^{0}+\frac{\partial \vec{f}^{0}}{\partial \lambda_{0}}\right),
$$

où

$$
\begin{aligned}
& \vec{f}^{0}=\vec{f}\left(x, \vec{\varphi}^{0}, \lambda_{0}\right), \\
& \vec{f}=\vec{f}\left(x, \vec{\varphi}^{0}+\left(\lambda-\lambda_{0}\right)\left(\vec{\psi}^{0}+\vec{v}\right), \lambda\right) .
\end{aligned}
$$

Posons

$$
\begin{aligned}
& \vec{w}=\overrightarrow{\phi^{0}}(x)+\vec{\chi}(x, \lambda), \\
& \vec{f}\left(x, \vec{\varphi}^{0}(x)+\left(\lambda-\lambda_{0}\right) \vec{w}, \lambda\right)=\vec{f}^{0}+\left(\lambda-\lambda_{0}\right)\left\{\sum_{k=1}^{n} \frac{\partial \vec{f}^{0}}{\partial \varphi_{k}^{0}} w_{k}\right. \\
& \left.\quad+\frac{\vec{f}^{0}}{\partial \lambda_{0}}+\vec{d}(x, \vec{w}, \lambda)\right\} .
\end{aligned}
$$


On voit, en remarquant $S \overrightarrow{(w)} \leqq \eta(x)$, que $\vec{\Delta}(x, \vec{w}, \lambda)$ converge uniformément vers $\overrightarrow{0}$ pour $\alpha \leqq x \leqq \beta(a<\alpha<\beta<b)$ lorsque $\lambda \rightarrow \lambda_{0}$. On peut donc faire correspondre à un nombre positif $\varepsilon$ un autre nombre positif $\delta$ de manière que l'on ait

$$
S(\vec{\Delta}(x, \vec{w}, \lambda))<\varepsilon
$$

pour

$$
\alpha \leqq x \leqq \beta, \quad\left|\lambda-\lambda_{0}\right| \leqq \delta, \quad \underline{\lambda} \leqq \lambda \leqq \bar{\lambda} .
$$

L'équation en $\vec{v}=\vec{\chi}(x, \lambda)$ peut s'écrire

$$
\overrightarrow{v^{\prime}}=\sum_{k=1}^{n} \frac{\partial \vec{f}^{0}}{\partial \varphi_{k}^{0}} v_{k}+\vec{\Delta}(x, \vec{w}, \lambda)
$$

Le second membre satisfait à l'inégalité

$$
S\left(\sum_{k=1}^{n} \frac{\partial \vec{f}^{0}}{\partial \varphi_{k}^{0}} v_{k}+\vec{\Delta}(x, \vec{w}, \lambda)\right) \leqq p(x) S(\vec{v})+\varepsilon .
$$

Par suite, on obtient, en appliquant le théorème de comparaison,

$$
S(\vec{\chi}(x, \lambda)) \leqq(1+K) \eta(\alpha) e^{P(x)-P(\alpha)}+\varepsilon e^{P(x)} \int_{\alpha}^{x} e^{-P(x)} d x
$$

dans l'intervalle $\alpha \leqq x \leqq \beta . \quad \varepsilon$ pouvant être aussi petit que l'on veut, on a

$$
\begin{aligned}
\varlimsup_{\lambda \rightarrow \lambda_{0}} S(\vec{\chi}(x, \lambda)) & \leqq(1+K) \eta(\alpha) e^{P(x)-P(\alpha)} \\
& =(1+K) e^{P(x)} \int_{a}^{\alpha} q(\xi) e^{-P(\xi)} d \xi .
\end{aligned}
$$

En y faisant $\alpha \rightarrow a+0$, on obtient

$$
\lim _{\lambda \rightarrow \lambda_{0}} S(\vec{\chi}(x, \lambda))=0 . \quad \text { C. Q. F. D. }
$$

Si le second membre $\vec{f}(x, \vec{y}, \lambda)$ contient $m$ paramètres $t_{1}, t_{2} \ldots$, $t_{m}$ et est continu par rapport à $(x, \vec{y}, \lambda, \mathbf{t})$ pour

$$
a<x<b, \quad S(\vec{y}) \leqq \omega(x), \quad \underline{\lambda} \leqq \lambda \leqq \bar{\lambda}, \mathrm{t} \in T,
$$

le second membre de (29) est continu par rapport à $(x, \vec{z}, \lambda, \mathbf{t})$ pour 


$$
a<x<b, \quad S\left(\vec{\varphi}^{0}+\left(\lambda-\lambda_{0}\right) \vec{z}\right) \leqq \omega(x), \quad \lambda \leqq \lambda \leqq \bar{\lambda}\left(\lambda \neq \lambda_{0}\right), \quad \mathbf{t} \in T .
$$

On voit, en appliquant le théorème 21, que la solution (31) est continue pár rapport à $(x, \lambda, \mathbf{t})$ pour

$$
a<x<b, \underline{\lambda} \leqq \lambda \leqq \bar{\lambda}\left(\lambda=\lambda_{0}\right), \mathbf{t} \in T .
$$

Si donc $\vec{\varphi}\left(x, \lambda_{\jmath}\right)$ est continue par rapport à $(x, \mathbf{t})$ pour $a<x<b$, $\mathbf{t} \in T, \overrightarrow{\varphi^{\prime}}(x, \lambda)$ est continue. par rapport à $(x, \lambda, \mathbf{t})$ pour (37). Prenons une valeur $\lambda_{1}$ appartenant à l'intervalle $\underline{\lambda} \leqq \lambda \leqq \bar{\lambda}$ et différente de $\lambda_{0} . \quad \vec{\varphi}\left(x, \lambda_{1}\right)$ étant continue pour $a<x<b, \mathbf{t} \in T$, on voit comme plus haut que $\vec{\varphi}(x, \lambda, \mathbf{t})$ est continue par rapport à $(x, \lambda, \mathbf{t})$ pour

$$
a<x<b, \underline{\lambda} \leqq \lambda \leqq \bar{\lambda}\left(\lambda \neq \lambda_{1}\right), \quad \mathbf{t} \in T .
$$

Elle est donc continue pour

$$
a<x<b, \underline{\lambda} \leqq \lambda \leqq \bar{\lambda}, \mathbf{t} \in T .
$$

Si de plus $\vec{f}(x, \vec{y}, \lambda)$ et ses dérivées partielles par rapport à $\lambda, y_{1}, \ldots, y_{n}$ sont continues par rapport à $(x, \vec{y}, \lambda, \mathbf{t})$ pour (36), $\vec{h}_{k}(x, \lambda)$, et $\vec{h}(x, \lambda)$ sont continues par rapport à $(x, \lambda, \mathbf{t})$ pour $(38)$. On voit, en appliquant le théorème 21 , que $\vec{\varphi}_{\lambda}^{\prime}(x, \lambda)$ est aussi continue par rapport à $(x, \lambda, \mathbf{t})$ pour (38). Nous arrivons ainsi au

THÉORÈmE 23. Nous supposons remplies les hypothèses suivantes :

$1^{\circ} \quad S(\vec{y})$ est positive sauf pour $\vec{y}=\overrightarrow{0}$.

$2^{\circ} \quad \omega(x)(\geqq 0), p(x), q(x)$ sont continues pour $a<x<b$.

$3^{\circ} \quad$ La famille $\mathfrak{F}$ contient la fonction identiquement nulle.

$4^{\circ}$ Les fonctions

$$
\begin{aligned}
& \vec{f}(x, \vec{y}, \lambda, \mathbf{t}), \vec{f}_{\lambda}^{\prime}(x, \vec{y}, \lambda, \mathbf{t}), \\
& \frac{\partial}{\partial y_{j}} \vec{f}(x, \vec{y}, \lambda, \mathbf{t}) \quad(j=1,2, \ldots ., n)
\end{aligned}
$$

sont continues par rapport à $(x, \vec{y}, \lambda, \mathbf{t})$ pour (36) et satisfont à l'inégalité (26).

$5^{\circ} \quad$ L'équation

$$
\overrightarrow{y^{\prime}}=\vec{f}(x, \vec{y}, \lambda, \mathbf{t})
$$


admet quels que soient $\lambda(\underline{\lambda} \leqq \lambda \leqq \bar{\lambda})$ et $\mathbf{t}(\epsilon T)$, une solution $\vec{y}=$ $\vec{\varphi}(x, \lambda, \mathbf{t})$ satisfaisant à l'inégalité

$$
S(\vec{\varphi}(x, \lambda, \mathbf{t})) \leqq \omega(x)
$$

dans l'intervalle $a<x<b$ et telle que

$$
S\left(\frac{\vec{\varphi}\left(x, \lambda_{1}, \mathbf{t}\right)-\vec{\varphi}\left(x, \lambda_{2}, \mathbf{t}\right)}{\lambda_{1}-\lambda_{2}}\right)
$$

appartient à $\mathfrak{F}, \lambda_{1}$ et $\lambda_{2}$ désignant des nombres quelconques dans l'intervalle $\underline{\lambda} \leqq \lambda \leqq \bar{\lambda}$.

$6^{\circ} \vec{\varphi}\left(x, \lambda_{0}\right)$ est continue par rapport à $(x, \mathbf{t})$ pour $a<x<b$, $\mathbf{t} \epsilon T, \lambda_{0}$ étant une valeur déterminée dans l'intervalle $\underline{\lambda} \leqq \lambda \leqq \bar{\lambda}$.

$7^{\circ}$. L'équation (28) admet une solution et une seule $Y=\eta(x)$ appartenant à $\mathfrak{F}$.

Dans ces hypothèses, $\vec{\varphi}(x, \lambda, \mathbf{t})$ est continue par rapport à $(x, \lambda, \mathbf{t})$, ainsi que sa dérivée $\vec{\varphi}_{\lambda}^{\prime}(x, \lambda, \mathbf{t})$, pour

$$
a<x<b, \underline{\lambda} \leqq \lambda \leqq \bar{\lambda}, \mathbf{t} \in T .
$$

9. Analyticité. En remarquant que, si une fonction est dérivable par rapport à une variable complexe dans un certain domaine, elle est holomorphe par rapport à ce variable dans ce domaine, nous obtenons sans peine, en étendant le théorème 23 au cas de variables $y_{1}, \ldots, y_{n}, \lambda$ complexes, le

THÉORÈmE 24. Nous supposons remplies les hypothèses suivantes:

$1^{\circ} \quad S(\vec{y})$ est positive sauf pour $\vec{y}=\overrightarrow{0}$.

$2^{\circ} \quad \omega(x)(\geqq 0), p(x), q(x)$ sont continues pour $a<x<b$.

$3^{\circ}$ La famille $\widetilde{F}$ contient la fonction identiquement nulle.

$4^{\circ}$ Les fonctions

$$
\begin{aligned}
& \vec{f}(x, \vec{y}, \lambda, \mathbf{t}), \vec{f}_{\lambda}^{\prime}(x, \vec{y}, \lambda, \mathbf{t}), \\
& \frac{\partial}{\partial y_{j}} \vec{f}(x, \vec{y}, \lambda, \mathbf{t}) \quad(j=1,2, \ldots, n)
\end{aligned}
$$

sont continues par rapport à $(x, \vec{y}, \lambda, \mathrm{t})$ pour

$$
a<x<b, S(\vec{y}) \leqq \omega(x), \quad \lambda \in \bar{A}, \quad \mathbf{t} \in T
$$


et satisfont à l'inégalité (26), $A$ étant un domaine dans le plan de nombres complexes et $\bar{A}$ son ensemble de fermeture.

$5^{\circ}$ L'équation (39) admet, quels que soient $\lambda(\epsilon \bar{\Lambda})$ et $\mathbf{t}(\epsilon T)$ une solution $\vec{y}=\vec{\varphi}(x, \lambda, \mathrm{t})$ satisfaisant à l'inégalité (40) dans l'intervalle $a<x<b$ et telle que

$$
S\left(\frac{\vec{\varphi}\left(x, \lambda_{1}, \mathbf{t}\right)-\vec{\varphi}\left(x, \lambda_{2}, \mathbf{t}\right)}{\lambda_{1}-\lambda_{2}}\right)
$$

appartient à $\mathfrak{F}, \lambda_{1}$ et $\lambda_{2}$ désignant des nombres quelconques dans $\bar{\lambda}$.

$6^{\circ} \vec{\varphi}\left(x, \lambda_{0}, \mathbf{t}\right)$ est continue par rapport à $(x, \mathbf{t})$ pour $a<x<b$, $\mathbf{t} \in T, \lambda_{0}$ étant une valeur déterminée de $\bar{\Lambda}$.

$7^{\circ}$ L'équation (28) admet une solution et une seule $Y=\eta(x)$ appartenant à $\mathfrak{F}$. $\lambda$ pour

Dans ces hypothèses, $\vec{\varphi}(x, \lambda, \mathbf{t})$ est holomorphe par rapport à

$$
a<x<b, \lambda \in A, \mathbf{t} \in T
$$

et continue par rapport à $(x, \lambda, \mathbf{t})$, ainsi que sa dérivée $\vec{\varphi}_{\lambda}^{\prime}(x, \lambda, \mathbf{t})$, pour

$$
a<x<b, \quad \lambda \in \bar{\Lambda}, \quad \mathbf{t} \in T
$$

\section{COMPLÉMENTS AU PREMIER MÉMOIRE}

$1^{\circ}$ L'hypothèse du théorème $15 \mathrm{du}$ Premier Mémoire: $\omega(x)$ est minorante $\grave{a}$ droite, peut être remplacée par la suivante: $\omega(x)$ est quasi inférieure à droite.

En effet, si l'on avait $\varphi(\xi)<\omega^{(\xi)}(a<\xi<b)$, on pourrait trouver un nombre $c(a \leqq c<b)$ tel que $\left({ }^{7}\right) \quad \varphi(x)<\omega(x)$ pour $c<x \leqq \xi$ et $\varphi(c)=\omega(c)$. La fonction $\chi(x)$ définie par

$$
\chi(x)= \begin{cases}\varphi(x) & \text { pour } a<x \leqq c, \\ \omega(x) & \text { pour } c<x \leqq \xi\end{cases}
$$

serait quasi supérieure à gauche et appartiendrait à $\mathfrak{F}$. On pourrait montrer qu'il existe une solution $y(x)$ telle que $\varphi(x) \leqq y(x) \leqq \chi(x)$

(7) Si $\varphi(x)<\omega(x)$ pour $a<x<\xi$, on pose $c=a$. 
pour $a<x<\xi$ et $\varphi(\xi)<y(\xi)<\chi(\xi)$. L'équation admettrait alors deux solutions $\varphi(x)$ et $y(x)$ appartenant à $\mathfrak{F}$, contrairement à l'hypothèse.

' $2^{\circ}$ Quant au théorème 17, l'hypothèse suivante est inutile: l'équation (11) est régulière à droite.

Il suffit d'appliquer le théorème amélioré ci-dessus, en posant

$$
\bar{\omega}(x)=\max \{\varphi(x), \Phi(x)\} .
$$

$3^{\circ}$ La même hypothèse est inutile pour établir les théorèmes 18 et 19 , car on les obtient en appliquant le théorème 17 .

11 janvier 1941. 